\title{
Magnetic activity of six young solar analogues I. Starspot cycles from long-term photometry
}

\author{
S. Messina ${ }^{1, \star}$ and E. F. Guinan ${ }^{2}$ \\ 1 INAF-Catania Astrophysical Observatory, Via S. Sofia 78, 95123 Catania, Italy \\ 2 Dept. of Astronomy and Astrophysics, Villanova University, Villanova 19085, PA USA \\ e-mail: edward.guinan@villanova.edu
}

Received 27 May 2002 / Accepted 5 July 2002

\begin{abstract}
A long-term photometric monitoring of a selected sample of solar analogues has been carried out since early nineties as part of The Sun in time project, which is aimed at a multiwavelength study of stars with solar-like global properties, but with different ages and thus at different stages of their evolution. The extended time sequence of ground-based observations collected over more than a decade as part of this program has revealed the existence of starspot cycles. Also from these data it is possible to investigate surface differential rotation of the stars. In this paper we present the photometry collected to date and report on cycles search for a selected subsample of five young single G0-G5V stars with ages between $\simeq 130 \mathrm{Myr}$ and $700 \mathrm{Myr}$ : EK Dra, $\pi^{1} \mathrm{UMa}, \mathrm{HN}$ Peg, $\mathrm{k}^{1}$ Cet and BE Cet. Also we include in this study the Pleiades-age $(\simeq 130 \mathrm{Myr}) \mathrm{K} 0 \mathrm{~V}$ star DX Leo (HD 82443). All the cited stars show activity cycles whose period is, furthermore, the first determined from photometric data. They are compared to those activity cycles derived from CaII H\&K emission fluxes and differences are discussed. All the cycle periods, except for EK Dra, fit well the empirical relations with global stellar parameters derived from larger stellar samples. The following results are also inferred from the present study: $i$ ) the fastest rotating stars tend to have longer cycles; ii) the range in the observed cycle lengths seems to converge with stellar age from a maximum dispersion around the Pleiades' age towards the solar cycle value at the Sun's age; iii) the overall short- and long-term photometric variability increases with inverse Rossby number with very high correlation degree, indicating that the level of magnetic activity at least in photosphere is still controlled by the stellar rotation even on the longest time scales; iiii) the increase with inverse Rossby number of the long-term overall photometric variability seems to level off at the highest rotation rate, which may be interpreted as due to a saturation in the level of photospheric magnetic activity around the activity maximum.
\end{abstract}

Key words. stars: activity - stars: individuals: BE Cet, $\mathrm{k}^{1}$ Cet, $\pi^{1} \mathrm{UMa}$, EK Dra, HN Peg, DX Leo - stars: late-type stars: magnetic fields - stars: starspots

\section{Introduction}

Solar analogues, that is stars having global stellar properties (e.g. mass, $T_{\text {eff }}$ and $L / L_{\odot}$ ) similar to those of the Sun, but having different ages, are suitable to study the time evolution of the Sun's magnetic activity. With this aim The Sun in time project was started in 1990 (Dorren \& Guinan 1994), a coordinated multiwavelength study of nearby, single, solartype stars selected as proxies for the Sun at several stages in its evolution from the ZAMS (Zero Age Main Sequence) to the TAMS (Terminal Age Main Sequence). The observations include ground-based photoelectric photometry, UV, EUVE, and X-ray measurements. The aim of the project is to determine the evolution of the stellar atmosphere and to

Send offprint requests to: $\mathrm{S}$. Messina,

e-mail: sme@sunct.ct.astro.it

* Guest User, Canadian Astronomy Data Center, which is operated by the Dominion Astrophysical Observatory for the National Research Council of Canada's Herzberg Institute of Astrophysics. investigate activity cycles and differential rotation. The aim also includes a description of the evolution of the solar dynamo and solar magnetic activity and the extraction of the solar UV and X-ray fluxes through the Sun's post-ZAMS history (Dorren \& Guinan 1994; Guinan et al. 2001).

In the recent years the time sequences of photometric measurements collected within the program have become long enough to allow the study of the photospheric activity on a time basis comparable to the 11-yr solar cycle. Our investigation is presently focused on a first subsample of six stars selected from the whole sample: BE Cet, $\mathrm{k}^{1}$ Cet, $\pi^{1} \mathrm{UMa}$, EK Dra, HN Peg and DX Leo (see Table 1). In a series of two papers we will present the long-term photometry collected to date and the results obtained on activity cycles and the latitudinal differential rotation. The first paper of this series actually concerns the starspot cycles.

In the framework of the solar-stellar connection the study of long-term behaviour of magnetic activity in solar-like stars is important to better understand the solar dynamo mechanism 
which is thought to be at the basis of the overall solar magnetic activity. The availability of a sample of solar analogues selected at different stages of their evolution enables us to infer accurate and unbiased empirical relations between activity quantities, which in this context are the presence, length and amplitude of cycles and stellar properties such as rotation, age and convective envelope depth. The exclusion from the selected sample of binaries or multiple systems and of out-of main-sequence stars eliminates the problem of unknown dependencies on tidal interaction and on out-of main-sequence convective envelope properties which may play a relevant role in the resulting magnetic activity properties. Such empirical relations are remarkably important being a valuable test for current dynamo theories. The use of tracers of photospheric activity in this study is also remarkably important since it allows us to investigate the evolution of the long-term activity up to the highest rotation rates and to investigate the temporal correlation of activity between the photosphere and the upper atmospheric layers.

\section{The sample}

\section{1. $H D 1835=B E$ Cet}

BE Cet $\left(V=+6.36, B-V=+0.66, P_{\text {rot }} \simeq 7.75^{\mathrm{d}}\right)$ is a G2 V single star with constant radial velocity $V_{\mathrm{r}}=-2.6 \mathrm{~km} \mathrm{~s}^{-1}$ (Gaidos et al. 2000). On the basis of its galactic space-velocity components it is classified as a member of the Hyades moving group with an estimated age of $\simeq 600 \mathrm{Myr}$. Its photometric variability with a period $P=7.655^{\mathrm{d}}$ was discovered by Chugainov (1980). BE Cet has been observed photometrically by a number of astronomers, Lockwood et al. (1997), Cutispoto (1991, 1992, 1995), Stepien \& Geyer (1996), Gaidos et al. (2000) and by the Hipparcos satellite (Perryman et al. 1997). BE Cet is among the stars observed at the Mt. Wilson Observatory as part of the CaII $H K$ project. Its chromospheric CaII H\&K emission fluxes vary with a cycle of $P_{\text {cyc }}=9.1 \mathrm{yr}$ (Baliunas et al. 1995). Within The Sun in time project, it was observed by ROSAT PSPC as part of the Guest Observer program (Guedel et al. 1997).

\section{2. $H D 20630=k^{1}$ Cet}

$\mathrm{k}^{1}$ Cet $\left(V=+4.80, B-V=+0.68, P_{\text {rot }} \simeq 9.21^{\mathrm{d}}\right)$ is a single G5 V star with constant radial velocity $V_{\mathrm{r}}=+18.9 \mathrm{~km} \mathrm{~s}^{-1}$ (Gaidos et al. 2000). On the basis of rotation-age relationship (Guedel et al. 1997), it is a likely member of the Hyades moving group with an estimated age of $\simeq 750 \mathrm{Myr}$. Its photometric variability was first detected with a period of $P=9.09^{\mathrm{d}}$ by the Hipparcos satellite. Since 1993 it has been photometrically monitored by Gaidos et al. (2000). $\mathrm{k}^{1}$ Cet has also variable CaII H\&K emission fluxes with a cycle of $P_{\text {cyc }}=5.6 \mathrm{yr}$ and a longer-term trend (Baliunas et al. 1995). Within The Sun in time project also $\mathrm{k}^{1}$ Cet was observed by ROSAT PSPC and ASCA as part of Guest Observer program (Guedel et al. 1997). Also observations have been carried out with IUE, EUVE and more recently with FUSE (see Guinan et al. 2001).

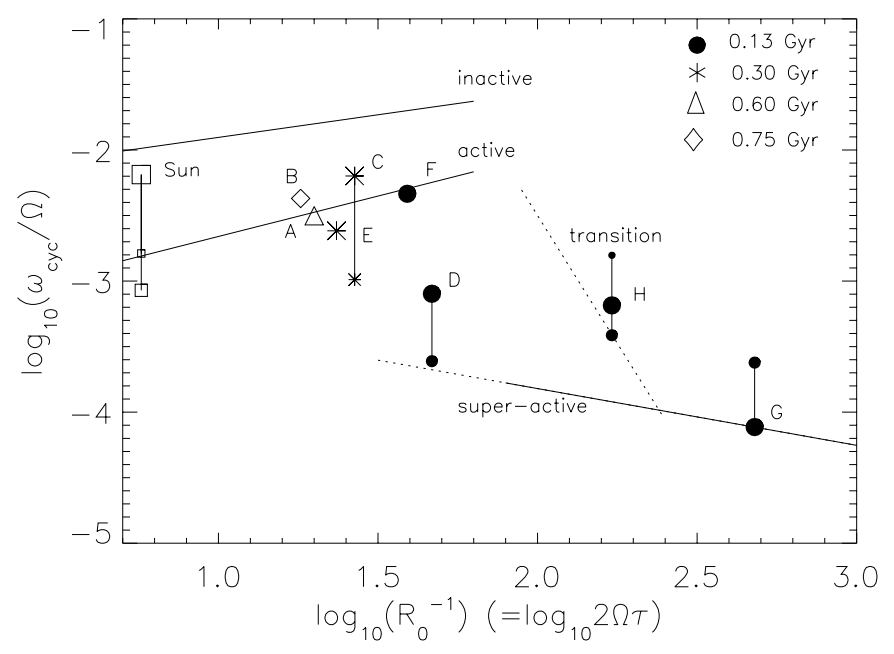

Fig. 1. $\log \left(\omega_{\text {cyc }} / \Omega\right)$ vs. $\log \left(R_{0}^{-1}\right)$ for the program stars. The cycles of LQ Hya $(G), A B$ Dor $(H)$ and the Sun are also plotted. Different stellar ages are indicated by different symbols, while each target is identified by the corresponding label as reported in Table 1. Smaller symbols denote secondary cycles. Lines are the least-squares fits from Saar \& Brandenburg (1999).

\section{3. $H D 72905=\pi^{1}$ UMa}

$\pi^{1} \mathrm{UMa}\left(V=+5.63, B-V=+0.62, P_{\text {rot }} \simeq 4.90^{d}\right)$ is a G1.5 V single star with constant radial velocity $V_{\mathrm{r}}=-12.7 \mathrm{~km} \mathrm{~s}^{-1}$ (Gaidos et al. 2000). It is a member of the Ursa Major moving group with an estimated age of $\simeq 300 \mathrm{Myr}$. Its photometric variability was discovered within the The Sun in time project (Guinan \& McCook 1991). It has been observed by Hipparcos and by Gaidos et al. (2000). Preliminary results of the current photometric monitoring were presented by Bochanski et al. (2001). $\pi^{1}$ UMa has variable CaII H\&K emission fluxes but no apparent cyclic behaviour (Baliunas et al. 1995). It was observed by ROSAT PSPC within the Guest Observer program (Guedel et al. 1997). Also, as part of the The Sun in time project, observations have been made with IUE, EUVE and FUSE (see Guinan et al. 2001).

\section{4. $H D 129333=E K$ Dra}

EK Dra $\left(V=+7.51, B-V=+0.61, P_{\text {rot }}=2.49-2.89^{\mathrm{d}}\right)$ is a G0 V star with constant radial velocity $V_{\mathrm{r}}=-30.5 \mathrm{~km} \mathrm{~s}^{-1}$ (Wilson 1953). On the basis of its galactic space-velocity components it is classified as a member of the Pleiades moving group with an estimated age of $\simeq 130 \mathrm{Myr}$. Although it may be a member of a wide binary system (Duquennoy \& Mayor 1994), because of its very long orbital period $(\simeq 12.5 \mathrm{yr})$, it can be considered as a single star as concerns its magnetic activity. It has been observed photometrically by Dorren \& Guinan (1994), Lockwood et al. (1997), Strassmeier et al. (1997, 1999) and by Hipparcos. EK Dra is reported by Baliunas et al. (1995) to be variable in the CaII H\&K emission fluxes with a longterm trend but with no apparent periodicity. Within The Sun in time project it was observed by ROSAT PSPC, ASCA and EUVE as part of Guest Observer program (Guedel et al. 1997) which detected high levels of X-ray and EUV emission 


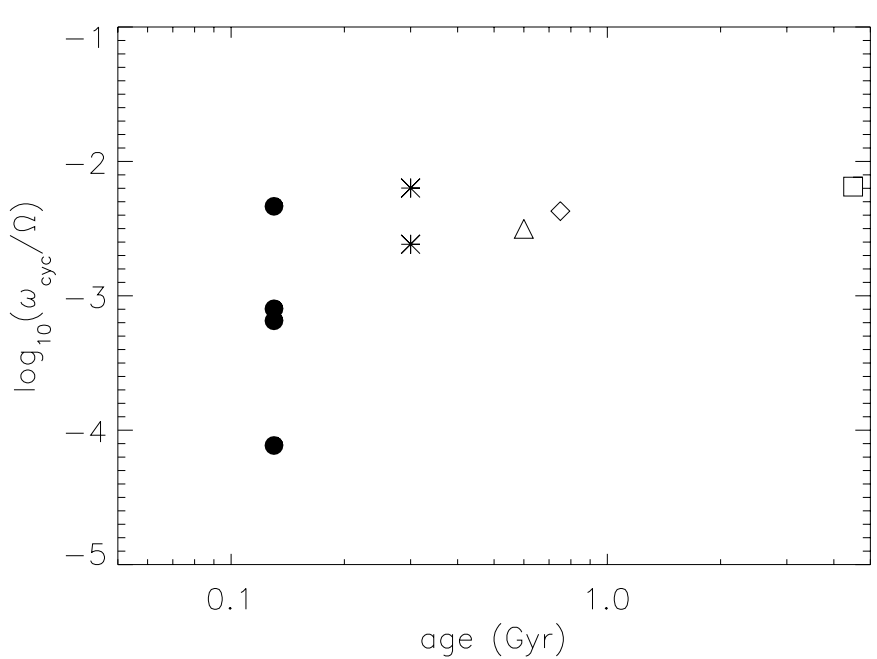

Fig. 2. $\log \left(\omega_{\text {cyc }} / \Omega\right)$ vs. age for the program stars. Symbols have the same meaning as in Fig. 1. The observed range in the primary cycles of single stars monotonically decreases from Pleiades age to the Sun's age.

including numerous flares. More recently, EK Dra has been observed with HST, Chandra and FUSE as part of this project.

\section{5. $H D 206860=H N P e g$}

HN Peg $\left(V=+5.94, B-V=+0.59, P_{\text {rot }} \simeq 4.64^{\mathrm{d}}\right)$ is a G0 V star with constant radial velocity $V_{\mathrm{r}}=-17.0 \mathrm{~km} \mathrm{~s}^{-1}$ (Gaidos et al. 2000). On the basis of age-rotation relashionships, it is a likely member of the Ursa Major moving group with an estimated age of $\simeq 300$ Myr. Its photometric variability was discovered by Blanco et al. (1972, 1979). It was photometrically observed by Reglero et al. (1986), Frasca et al. (2000), Gaidos et al. (2000) and Hipparcos. HN Peg has a CaII chromospheric cycle of $P_{\text {cyc }}=6.2 \mathrm{yr}$ and a longer-term trend (Baliunas et al. 1995). Within The Sun in time project it was observed by ASCA as part of Guest Observer program (Guedel et al. 1997) and also with IUE and EUVE.

\section{6. $H D 82443=D X$ Leo}

DX Leo $\left(V=+7.01, B-V=+0.76, P_{\text {rot }} \simeq 5.42^{\mathrm{d}}\right)$ is a single $\mathrm{K} 0 \mathrm{~V}$ star with constant radial velocity $V_{\mathrm{r}}=+8.2 \mathrm{~km} \mathrm{~s}^{-1}$ (Gaidos et al. 2000). On the basis of its galactic space-velocity components it is classified as a member of the Pleiades moving group with an estimated age of $\simeq 130 \mathrm{Myr}$. Its photometric variability was discovered as part of the The Sun in time project (Guinan \& McCook 1991). It has been observed photometrically by Henry et al. (1995) who first reported a period of $P=5.43^{\mathrm{d}}$. DX Leo has been observed also by Gaidos et al. (2000), Strassmeier et al. $(1997,1999)$ and by Hipparcos. The presence of a starspot cycle and of surface differential rotation were first inferred by a previous study based on the first ten years of the current photometric monitoring (Messina et al. 1999). Within the $H K$ project DX Leo was found to show a CaII chromospheric activity cycle of $P_{\text {cyc }} \simeq 2.8 \mathrm{yr}$ (Baliunas et al. 1995).

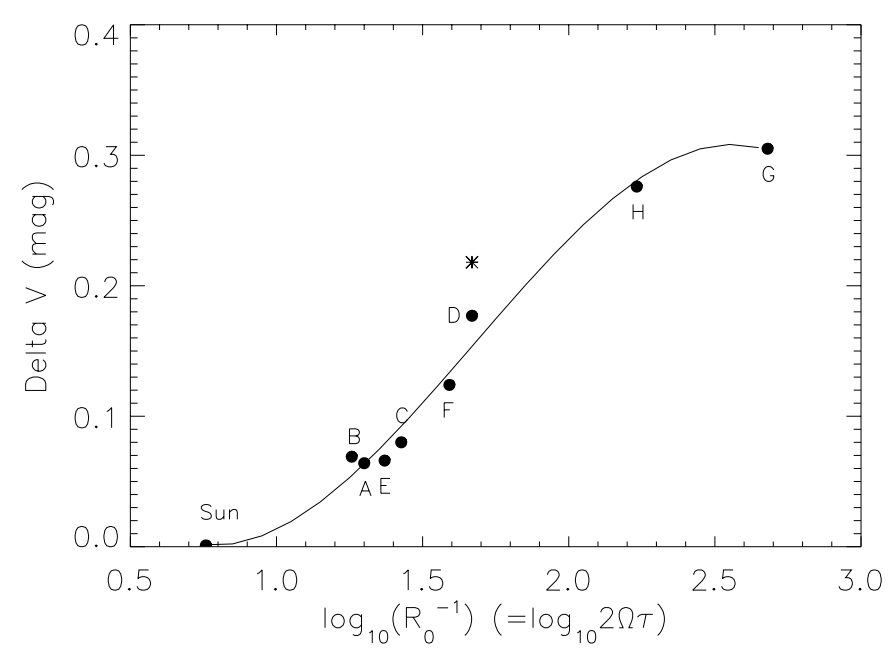

Fig. 3. The amplitude of the overall $V$-band variability is plotted vs. $\log \left(R_{0}^{-1}\right)$. Continuous line represents a cubic fit to the data. The amplitude is computed as the difference between the brightest and faintest observed magnitudes. In the case of EK Dra (D) the filled dot denotes the only cycle amplitude, while the asterisk denotes cycle amplitude plus longer-term trend.

\section{Observations}

\subsection{New photometry}

The new photoelectric observations presented in this paper were obtained, from 1988 through 2000, with three different Automatic Photoelectric Telescopes (APTs): a) the 0.25-m T1 Phoenix APT of Fairborn Observatory in southern Arizona and managed as multiuser telescope (Boyd et al. 1984; Seeds 1995); b) the 0.75-m T5 APT; c) the 0.75-m T6 APT telescopes both operated at the Fairborn Observatory (AZ, USA) for Villanova University, as part of the Four College Consortium ${ }^{1}$.

The observations were made in the years from 1988 to 1989 by the $0.25-\mathrm{m}$ Phoenix telescope equipped with standard Johnson $U B V$ filters and feeding an uncooled 1P21 photomultiplier. In 1990 the program stars were transfered to the Fairborn T5 and T6 APTs equipped with standard $U B V(R I)_{\mathrm{C}}$ filters matching the Johnson-Cousins system (Bessel 1990) and feeding temperature controlled EMI-9828 photomultipliers. EK Dra and HN Peg were observed with uvby filters matching the Strömgren system (Strömgren 1966).

All the observations were made differentially with respect to suitable comparison (c) and check (ck) stars (Table 2). Tensecond integrations were used in each filter and the observing sequence $n-c-c k-c-v-v-c-s$ etc., where the symbol $v$ denotes the program star and $n$ the bright navigation star, which is the first star of the sequence the APT searches. The sky background (s) was measured at a fixed position near each star.

The observations were corrected for sky-background and atmospheric extinction and the instrumental differential magnitudes were transformed into the standard photometric systems.

\footnotetext{
1 In early 1996 the T6 telescope was acquired by the University of Vienna. In late 1996 all the telescopes operated by Fairborn were relocated from Mt. Hopkins, AZ close to Washington Camp (AZ, USA).
} 
Table 1. Stellar properties for the program stars and for three additional stars not observed as part of The Sun in time project but with known photometric starspot cycles. The starspot cycle periods $\left(P_{\text {cyc }}\right)$ for the program stars are listed with corresponding uncertainty $(\Delta P)$ and falsealarm-probability $(F A P)$. The labels are given to identify the stars in Fig. 1 and in Fig. 3.

\begin{tabular}{|c|c|c|c|c|c|c|c|c|c|c|c|c|}
\hline $\begin{array}{l}\text { HD } \\
\text { number }\end{array}$ & Name & Sp. T. & $\begin{array}{c}V \\
(\mathrm{mag})\end{array}$ & $\begin{array}{l}B-V \\
\text { (mag) }\end{array}$ & $\begin{array}{l}\tau_{\mathrm{c}} \\
(\mathrm{d})\end{array}$ & $\begin{array}{r}P_{\text {rot }} \\
\text { (d) }\end{array}$ & $\frac{P_{\text {cyc1 }} \pm \Delta P}{(\mathrm{yr})}$ & $F A P$ & $\frac{P_{\mathrm{cyc} 2} \pm \Delta P}{(\mathrm{yr})}$ & $F A P$ & $\frac{P_{\mathrm{cyc} 3} \pm \Delta P}{(\mathrm{yr})}$ & Label \\
\hline
\end{tabular}

\begin{tabular}{|c|c|c|c|c|c|c|c|c|c|c|c|c|}
\hline \multicolumn{13}{|c|}{ Program stars } \\
\hline 1835 & BE Cet & $\mathrm{G} 2 \mathrm{~V}$ & 6.36 & 0.66 & 12.33 & 7.756 & $6.7 \pm 0.7$ & $4.10 \mathrm{e}-1$ & - & - & - & A \\
\hline 20630 & $\mathrm{k}^{1}$ Cet & G5 V & 4.80 & 0.68 & 13.27 & 9.214 & $5.9 \pm 0.2$ & $7.38 \mathrm{e}-3$ & - & - & - & B \\
\hline 72905 & $\pi^{1} \mathrm{UMa}$ & $\mathrm{G} 1.5 \mathrm{~V}$ & 5.63 & 0.62 & 10.43 & 4.905 & $13.1 \pm 0.9$ & $2.05 \mathrm{e}-4$ & $2.12 \pm 0.05$ & $5.15 e-2$ & - & $\mathrm{C}$ \\
\hline 129333 & EK Dra & G0 V & 7.51 & 0.61 & 9.96 & 2.682 & $9.2 \pm 0.4$ & $3.46 \mathrm{e}-6$ & $>30$ & - & - & D \\
\hline 206860 & HN Peg & G0 V & 5.94 & 0.59 & 9.03 & 4.84 & $5.5 \pm 0.3$ & $5.57 \mathrm{e}-6$ & - & - & - & $\mathrm{E}$ \\
\hline \multirow[t]{2}{*}{82443} & DX Leo & K0 V & 7.01 & 0.76 & 16.86 & 5.424 & $3.21 \pm 0.05$ & $6.87 \mathrm{e}-7$ & - & - & - & $\mathrm{F}$ \\
\hline & & & \multicolumn{10}{|c|}{ Additional single stars with known photometric starspots cycles } \\
\hline 36705 & AB Dor & $\mathrm{K} 0 \mathrm{~V}$ & 6.89 & 0.83 & 19.61 & 0.5144 & $18.30^{a}$ & - & $5.9^{a}$ & - & - & G \\
\hline 82558 & LQ Hya & $\mathrm{K} 2 \mathrm{~V}$ & 7.74 & 0.90 & 21.87 & 1.61 & $6.75^{b}$ & - & $11.40^{b}$ & - & $2.8^{b}$ & $\mathrm{H}$ \\
\hline & Sun & $\mathrm{G} 2 \mathrm{~V}$ & - & 0.66 & 11.9 & 26.09 & 10.5 & - & 84 & - & 44 & \\
\hline
\end{tabular}

${ }^{a}$ Messina et al. in progress; ${ }^{b}$ Oláh et al. (2000).

The transformation into the standard system is made with an accuracy of the order of $0.01 \mathrm{mag}$ in the $V$ magnitude. No significant light variation was detected from the differential measures of comparison and check stars, indicating that the light of the comparison star is constant within about \pm 0.005 mag. Due to the relatively short duration of an observing sequence ( $\simeq 20 \mathrm{~min}$ ), the $v-c$ values were averaged to obtain one single data point for each night. The typical standard deviation of the averaged $V$-band $v-c$ magnitudes is of the order of 0.007 mag.

The $y$ magnitudes in the Strömgren system obtained for EK Dra and HN Peg were transformed into Johnson $V$ magnitudes according to Olsen (1983). Such transformation was necessary in order to compare our data with the historical $V$-band light curves from the literature.

\subsection{Historical photometry}

All the light curves available in the literature were collected and consistently analysed to investigate the variability on the longest possible time baselines.

In the following analysis we have considered only $V$-band light curves because they represent a more complete and homogeneous data set. The less numerous $U, B, R, I$ (and $u, v, b$ for EK Dra and HN Peg) light curves do not add any independent information for the cycle and surface differential rotation detection and analysis.

Differential photometry of each program star was obtained in the past sometimes using different comparison stars. When the same comparison star was employed, different values of its $V$-band standard magnitude were sometimes used to transform differential values into standard magnitudes. To obtain a homogeneous data set all the measurements from the literature were converted into differential values with respect to the same comparison star as listed in Table 2. $\Delta y$ magnitudes were also converted into the Johnson $V$ magnitudes according to
Table 2. Comparison and check stars used for the differential photometry of the program stars. $V_{\text {comp }}$ is the magnitude of the comparison star which is adopted to convert differential measures into absolute values.

\begin{tabular}{lllll}
\hline \hline \multicolumn{2}{c}{ Program star (v) } & Comparison (c) & Check (ck) & $V_{\text {comp }}$ \\
\hline HD 1835 $=$ BE Cet & HD 1388 & HD 645 & 6.50 \\
HD 20630 $=\mathrm{k}^{1}$ Cet & HD 20791 & HD 21585 & 5.70 \\
HD 72905 $=\pi^{1}$ UMa & HD 73108 & HD 72037 & 4.60 \\
HD 82443 = DX Leo & HD 82191 & HD 81146 & 6.64 \\
HD 129333 = EK Dra & HD 129390 & HD 127821 & 7.55 \\
HD 206860 = HN Peg & HD 207223 & HD 207652 & 6.20 \\
\hline
\end{tabular}

Olsen (1983). However, the conversion of $\Delta y$ to $\Delta V$ involved only a small correction.

To guarantee that all the light curves be comparable with each other and with the newly presented photometry (hereafter referred to as Villanova photometry) we applied corrections to minimize the systematic differences among light curves observed at the same epoch. Such systematic differences never exceeded $0.015 \mathrm{mag}$ and were applied to all the light curves from the same source. Finally, after having recalibrated all the measurements in a consistent way, the differential magnitudes were converted into the standard magnitude scale adopting for the comparison star the values of $V_{\text {comp }}$ in Table 2. As a result of the procedure described above, we believe that the standard magnitude values of the whole photometric time series are consistent up to the 0.01 mag level.

The $H_{\mathrm{p}}$ magnitudes of the Hipparcos satellite were transformed into Johnson $V$ magnitudes using the $H_{\mathrm{p}}-V$ versus $V-I$ relation taken from Perryman et al. (1997) and adopting for each star the appropriate $V-I$ value provided by Hipparcos.

High precision photoelectric photometry for all the program stars, except for EK Dra, was taken from Gaidos et al. (2000). Photometry for BE Cet was taken from 
Table 3. BE Cet: Mid epoch (HJD-2440000), time range, number of data points, mean magnitude $\left(V_{\max }+V_{\min }\right) / 2$ and sources of the light curves.

\begin{tabular}{|c|c|c|c|c|c|c|c|c|c|}
\hline Mid epoch & HJD range & $N_{\mathrm{m}}$ & $V_{\text {mean }}$ & Source & Mid epoch & HJD range & $N_{\mathrm{m}}$ & $V_{\text {mean }}$ & Source \\
\hline 1986.89 & $6736.72-6745.62$ & 9 & 6.397 & $\mathrm{a}$ & 1993.71 & $9234.80-9263.74$ & 24 & 6.388 & $\mathrm{f}, \mathrm{g}$ \\
\hline 1986.85 & $6754.66-6761.25$ & 6 & 6.397 & $\mathrm{a}$ & 1993.81 & $9270.74-9295.71$ & 18 & 6.387 & $\mathrm{f}, \mathrm{g}$ \\
\hline 1987.93 & $7129.62-7143.59$ & 9 & 6.390 & $\mathrm{~b}$ & 1993.92 & $9297.68-9353.61$ & 27 & 6.389 & $\mathrm{f}$ \\
\hline 1989.07 & $7549.55-7557.55$ & 8 & 6.377 & $\mathrm{c}$ & 1994.82 & $9612.79-9689.62$ & 45 & 6.386 & $\mathrm{f}$ \\
\hline 1989.95 & 7869.61-7876.61 & 7 & 6.383 & d & 1995.77 & 9986.77-10013.75 & 14 & 6.377 & $\mathrm{~g}$ \\
\hline 1990.44 & 8029.89-8078.06 & 12 & 6.385 & $\mathrm{e}$ & 1995.95 & $10035.72-10092.59$ & 10 & 6.385 & $\mathrm{~g}$ \\
\hline 1990.90 & $8200.77-8245.57$ & 16 & 6.385 & $\mathrm{f}$ & 1996.92 & $10396.62-10441.65$ & 16 & 6.382 & $\mathrm{f}$ \\
\hline 1991.07 & $8280.57-8290.00$ & 17 & 6.381 & $\mathrm{e}$ & 1997.80 & 10714.80-10768.69 & 36 & 6.375 & $\mathrm{f}, \mathrm{g}$ \\
\hline 1991.80 & $8536.73-8559.67$ & 12 & 6.388 & $\mathrm{f}$ & 1998.83 & $11085.77-11148.65$ & 28 & 6.390 & $\mathrm{~g}$ \\
\hline 1991.89 & $8561.66-8606.66$ & 14 & 6.395 & $\mathrm{f}$ & 1998.99 & $11151.74-11197.58$ & 32 & 6.387 & $\mathrm{f}, \mathrm{g}$ \\
\hline 1992.79 & $8897.72-8928.67$ & 16 & 6.380 & $\mathrm{f}$ & 1999.77 & $11447.93-11472.84$ & 6 & 6.387 & $\mathrm{f}$ \\
\hline 1992.88 & $8930.67-8958.69$ & 15 & 6.383 & $\mathrm{f}$ & 2000.02 & $11543.62-11560.59$ & 10 & 6.385 & $\mathrm{f}$ \\
\hline
\end{tabular}

(a) Stepien \& Geyer (1996); (b) Cutispoto (1991); (c) Cutispoto (1992); (d) Cutispoto (1995); (e) Hipparcos;

(f) Villanova photometry; (g) Gaidos et al. (2000).

Stepien \& Geyer (1996) and Cutispoto (1991, 1992, 1995). For instance, we shifted the Stepien \& Geyer's $V$-band data by +0.03 mag because we adopted for the comparison star of BE Cet the value of $V_{\text {comp }}=6.87$ given by Cutispoto (1991).

The photometry of DX Leo taken in the 1988-1998 period was already presented by Messina et al. (1999). Additional photometry of DX Leo was taken from Strassmeier et al. $(1997,1999)$.

Additional sources of photometry for EK Dra were Lockwood et al. (1997) and Strassmeier et al. (1997, 1999). Photometry of HN Peg was retrieved from Blanco et al. (1979), Reglero et al. (1986) and Frasca et al. (2000). It is worth noting that the photometry obtained by Blanco et al. was shifted by $\Delta V=+0.03$ mag according to updated magnitudes for the standard stars they used to transform differential measurements into absolute values.

\section{Analysis}

It proved possible to select for each program star several time intervals (see Table 3 to Table 7) along which the flux showed a regular modulation which could be attributed to the rotation of a stationary spot pattern on the photosphere of the star. In this way, 24 light curves were obtained for BE Cet, 18 for $\mathrm{k}^{1}$ Cet, 35 for $\pi^{1} \mathrm{UMa}, 92$ for EK Dra, 12 for HN Peg and 53 for DX Leo. All the light curves have a good coverage in rotational phase and, therefore, provide a reliable indication of the star's maximum and minimum magnitude and of the peakto-peak amplitude at any given epoch. In several light curves, in spite of the good rotational phase coverage, data points are more clustered near to the light curve maximum or minimum. Consequently, the mean value between the maximum and minimum magnitudes $\left(V_{\max }+V_{\min }\right) / 2$ differs from the mean brightness when computed by averaging all the magnitudes. We believe that the first quantity (represented by open diamonds in Fig. 4 to Fig. 9) more reliably indicates the mean star's brightness at any given epoch. We decided to analyse the sequence of mean magnitudes instead of the whole sequence of nightly data points, in spite of the decreased accuracy in the cycle length determination because of the much smaller number of data points considered.

For most stars the presence of starspot cycles can be seen just from a visual inspection. Nonetheless, a Scargle-Press period search routine (Scargle 1982) was applied to the whole sequence of mean magnitudes and all the stars were found to have well defined starspot cycles. The results of the periodogram analysis are reported in Table 1 where we report the cycle period $\left(P_{\text {cyc } 1}\right)$ and its uncertainty $(\Delta P)(\mathrm{Col} .8)$, as derived by the method of Horne \& Baliunas (1986) and the falsealarm-probability (FAP) of the peak frequency (Col.9). This last quantity estimates the probability that a peak of that height could result from a similar sample of Gaussian noise with the same variance of the analysed data (cf. Horne \& Baliunas 1986).

A search for secondary periodicities was also performed by filtering the primary frequency modulation from the data and recomputing the periodogram for the residual data. The results are reported in Col. 10 of Table 1. For all the stars, except for $\pi^{1} \mathrm{UMa}$, only noise remained in the power spectrum after filtering the primary frequency. In the case of $\pi^{1} \mathrm{UMa}$ a period of $P=2.12$ yr with a $F A P \simeq 0.05$ and a power significantly higher than the noise level was detected. In the case of EK Dra, the detected 9.2-yr cycle is clearly superimposed on a longer term trend. However, this long-term trend may be a segment of cycle with a period much longer than the time extension of the available photometry (in this case longer than 30 years) and 
Table 4. $\mathrm{k}^{1}$ Cet.

\begin{tabular}{|c|c|c|c|c|c|c|c|c|c|}
\hline Mid epoch & HJD range & $N_{\mathrm{m}}$ & $V_{\text {mean }}$ & Source & Mid epoch & HJD range & $N_{\mathrm{m}}$ & $V_{\text {mean }}$ & Source \\
\hline 1990.36 & $7908.21-8135.62$ & 12 & 4.841 & $\mathrm{a}$ & 1995.75 & 9986.90-9998.90 & 14 & 4.845 & $\mathrm{~b}$ \\
\hline 1991.37 & $8256.23-8525.77$ & 37 & 4.851 & $\mathrm{a}$ & 1995.88 & 10004.88-10074.74 & 23 & 4.842 & $\mathrm{~b}$ \\
\hline 1992.59 & $8647.73-9031.42$ & 18 & 4.830 & $\mathrm{a}$ & 1996.06 & $10086.75-10123.64$ & 10 & 4.838 & b \\
\hline 1993.72 & 9239.92-9259.86 & 12 & 4.813 & $\mathrm{~b}$ & 1996.90 & $10391.93-10435.66$ & 17 & 4.842 & $\mathrm{~b}, \mathrm{c}$ \\
\hline 1993.81 & $9268.87-9301.90$ & 31 & 4.822 & $\mathrm{~b}, \mathrm{c}$ & 1997.04 & $10443.77-10482.70$ & 13 & 4.845 & $\mathrm{~b}, \mathrm{c}$ \\
\hline 1993.92 & 9312.67-9338.73 & 23 & 4.819 & $\mathrm{~b}, \mathrm{c}$ & 1997.79 & 10718.95-10755.92 & 14 & 4.843 & $\mathrm{~b}$ \\
\hline 1994.04 & 9346.61-9393.66 & 17 & 4.816 & $\mathrm{~b}, \mathrm{c}$ & 1997.95 & 10759.90-10836.71 & 11 & 4.838 & $\mathrm{~b}$ \\
\hline 1994.78 & $9624.92-9652.95$ & 18 & 4.818 & $\mathrm{~b}, \mathrm{c}$ & 1998.84 & $11102.94-11142.83$ & 19 & 4.829 & $\mathrm{~b}$ \\
\hline 1994.99 & $9653.76-9773.60$ & 40 & 4.821 & $\mathrm{~b}, \mathrm{c}$ & 1999.09 & 11188.76-11240.61 & 12 & 4.825 & b \\
\hline
\end{tabular}

(a) Hipparcos; (b) Gaidos et al. (2000); (c) Villanova photometry.

Table 5. $\pi^{1}$ UMa.

\begin{tabular}{lcrrllcccc}
\hline \hline Mid epoch & HJD range & $N_{\mathrm{m}}$ & $V_{\text {mean }}$ & Source & Mid epoch & HJD range & $N_{\mathrm{m}}$ & $V_{\text {mean }}$ & Source \\
\hline 1989.30 & $7625.66-7650.69$ & 14 & 5.677 & $\mathrm{a}$ & 1995.18 & $9755.70-9808.77$ & 19 & 5.660 & $\mathrm{~b}$ \\
1989.87 & $7815.03-7874.05$ & 13 & 5.667 & $\mathrm{a}$ & 1995.94 & $10029.95-10089.86$ & 28 & 5.643 & $\mathrm{~b}$ \\
1990.07 & $7908.92-7926.87$ & 5 & 5.671 & $\mathrm{a}$ & 1996.12 & $10096.88-10159.77$ & 23 & 5.643 & $\mathrm{a}, \mathrm{b}$ \\
1990.30 & $7991.71-8012.69$ & 3 & 5.679 & $\mathrm{a}$ & 1996.29 & $10160.71-10214.65$ & 40 & 5.652 & $\mathrm{a}, \mathrm{b}$ \\
1990.82 & $8174.98-8211.95$ & 13 & 5.671 & $\mathrm{a}$ & 1996.97 & $10405.99-10474.81$ & 32 & 5.653 & $\mathrm{a}, \mathrm{b}$ \\
1990.99 & $8230.83-8275.85$ & 14 & 5.682 & $\mathrm{a}$ & 1997.13 & $10479.87-10517.77$ & 34 & 5.652 & $\mathrm{a}, \mathrm{b}$ \\
1991.17 & $8301.79-8338.67$ & 11 & 5.682 & $\mathrm{a}$ & 1997.27 & $10525.68-10569.68$ & 12 & 5.656 & $\mathrm{~b}$ \\
1991.26 & $8340.68-8361.64$ & 15 & 5.672 & $\mathrm{a}$ & 1997.87 & $10736.02-10801.95$ & 20 & 5.636 & $\mathrm{~b}$ \\
1992.10 & $8622.88-8697.67$ & 20 & 5.682 & $\mathrm{a}$ & 1998.07 & $10806.94-10876.73$ & 46 & 5.635 & $\mathrm{a}, \mathrm{b}$ \\
1992.27 & $8699.75-8742.64$ & 11 & 5.678 & $\mathrm{a}$ & 1998.21 & $10872.80-10908.67$ & 13 & 5.635 & $\mathrm{a}, \mathrm{b}$ \\
1993.14 & $9018.75-9062.71$ & 14 & 5.665 & $\mathrm{a}$ & 1998.32 & $10910.71-10949.65$ & 19 & 5.637 & $\mathrm{a}, \mathrm{b}$ \\
1993.86 & $9284.98-9317.01$ & 28 & 5.646 & $\mathrm{a}, \mathrm{b}$ & 1998.91 & $11118.97-11176.80$ & 18 & 5.651 & $\mathrm{~b}$ \\
1993.98 & $9331.83-9359.94$ & 24 & 5.654 & $\mathrm{a}, \mathrm{b}$ & 1999.08 & $11175.78-11240.79$ & 23 & 5.653 & $\mathrm{~b}$ \\
1994.07 & $9362.82-9398.82$ & 26 & 5.651 & $\mathrm{a}, \mathrm{b}$ & 1999.26 & $11247.81-11296.64$ & 29 & 5.649 & $\mathrm{a}, \mathrm{b}$ \\
1994.26 & $9411.73-9482.65$ & 34 & 5.642 & $\mathrm{a}, \mathrm{b}$ & 1999.36 & $11300.69-11317.66$ & 8 & 5.658 & $\mathrm{~b}$ \\
1994.87 & $9645.00-9696.86$ & 23 & 5.652 & $\mathrm{~b}$ & 2000.05 & $11546.81-11582.77$ & 16 & 5.652 & $\mathrm{a}, \mathrm{b}$ \\
1994.99 & $9701.84-9724.93$ & 20 & 5.653 & $\mathrm{a}, \mathrm{b}$ & 2000.22 & $11612.74-11634.68$ & 9 & 5.652 & $\mathrm{a}, \mathrm{b}$ \\
1995.07 & $9731.76-9754.82$ & 28 & 5.656 & $\mathrm{a}, \mathrm{b}$ & & & & &
\end{tabular}

(a) Villanova photometry; (b) Gaidos et al. (2000).

Table 6. HN Peg.

\begin{tabular}{llllllllll}
\hline \hline Mid epoch & HJD range & $N_{\mathrm{m}}$ & $V_{\text {mean }}$ & Source & Mid epoch & HJD range & $N_{\mathrm{m}}$ & $V_{\text {mean }}$ & Source \\
\hline 1990.66 & $7901-8249$ & 20 & 5.979 & $\mathrm{a}$ & 1994.85 & $9633-9706$ & 37 & 5.975 & $\mathrm{~b}$ \\
1991.57 & $8363-8565$ & 20 & 5.978 & $\mathrm{a}$ & 1995.45 & $9862-9908$ & 33 & 5.978 & $\mathrm{~b}, \mathrm{c}$ \\
1992.43 & $8758-8806$ & 16 & 5.977 & $\mathrm{a}$ & 1995.84 & $9986-10071$ & 34 & 5.979 & $\mathrm{~b}, \mathrm{c}$ \\
1993.43 & $9126-9163$ & 22 & 5.972 & $\mathrm{~b}$ & 1996.90 & $10392-10443$ & 20 & 5.974 & $\mathrm{~b}$ \\
1993.82 & $9239-9337$ & 44 & 5.976 & $\mathrm{~b}$ & 1997.87 & $10714-10806$ & 33 & 5.974 & $\mathrm{~b}$ \\
1994.45 & $9491-9547$ & 18 & 5.976 & $\mathrm{~b}$ & 1998.76 & $11010-11179$ & 64 & 5.973 & $\mathrm{~b}, \mathrm{~d}$ \\
\hline
\end{tabular}

(a) Hipparcos; (b) Gaidos et al. (2000); (c) Villanova photometry; (d) Frasca et al. (2000).

not yet resolved by the time series. The cycle period determinations have an uncertainty due to the finite length of the time series. However, the active region growth and decay (ARGD) may be an additional source of indetermination as shown by e.g. Donahue et al. (1996). In the following paper of this series concerning the surface differential rotation detected in the program stars, the results of the pooled variance analysis (Dobson et al. 1990) will be presented, which show that the ARGD develops on a time scale well distincted from the starspot cycles length. 
Table 7. EK Dra.

\begin{tabular}{|c|c|c|c|c|c|c|c|c|c|}
\hline Mid epoch & HJD range & $N_{\mathrm{m}}$ & $V_{\text {mean }}$ & source & Mid epoch & HJD range & $N_{\mathrm{m}}$ & $V_{\text {mean }}$ & source \\
\hline 1984.38 & $5806.90-5873.73$ & 6 & 7.554 & $\mathrm{a}$ & 1994.34 & $9472.72-9481.72$ & 21 & 7.595 & $\mathrm{~b}, \mathrm{~d}$ \\
\hline 1985.36 & $6187.82-6206.73$ & 4 & 7.529 & $\mathrm{a}$ & 1994.37 & $9482.68-9493.80$ & 33 & 7.588 & $a, b, d$ \\
\hline 1985.43 & $6217.73-6225.75$ & 5 & 7.553 & $\mathrm{a}$ & 1994.42 & $9499.68-9513.79$ & 18 & 7.600 & $\mathrm{~b}, \mathrm{~d}$ \\
\hline 1985.48 & $6232.73-6250.70$ & 9 & 7.553 & $\mathrm{a}$ & 1994.45 & $9514.78-9520.77$ & 12 & 7.585 & $\mathrm{a}, \mathrm{b}$ \\
\hline 1986.17 & 6486.87-6495.93 & 10 & 7.566 & $\mathrm{a}$ & 1994.50 & 9526.68-9544.71 & 18 & 7.587 & $a, b, d$ \\
\hline 1986.22 & $6504.88-6519.88$ & 11 & 7.565 & $\mathrm{a}$ & 1994.97 & $9703.03-9715.00$ & 4 & 7.639 & $\mathrm{~b}$ \\
\hline 1986.35 & $6547.83-6568.79$ & 9 & 7.562 & $\mathrm{a}$ & 1995.01 & 9715.04-9731.99 & 3 & 7.592 & $\mathrm{~b}$ \\
\hline 1986.49 & $6593.73-6621.71$ & 13 & 7.580 & a & 1995.10 & 9750.90-9760.99 & 10 & 7.622 & $\mathrm{~b}$ \\
\hline 1987.29 & 6881.97-6919.77 & 7 & 7.591 & $\mathrm{a}$ & 1995.17 & 9772.88-9785.84 & 6 & 7.607 & $\mathrm{~b}$ \\
\hline 1987.47 & $6949.72-6982.73$ & 15 & 7.611 & $\mathrm{a}$ & 1995.26 & 9810.77-9817.76 & 7 & 7.610 & $\mathrm{~b}$ \\
\hline 1988.14 & 7198.96-7231.94 & 7 & 7.603 & $\mathrm{a}$ & 1995.45 & $9860.73-9904.71$ & 27 & 7.639 & $b, d$ \\
\hline 1989.30 & $7623.88-7646.78$ & 20 & 7.609 & $a, b$ & 1996.00 & $10075.01-10094.00$ & 40 & 7.627 & $\mathrm{~b}$ \\
\hline 1989.36 & $7647.78-7668.73$ & 12 & 7.613 & $a, b$ & 1996.04 & 10094.01-10100.98 & 24 & 7.626 & $\mathrm{~b}$ \\
\hline 1989.42 & $7670.73-7687.70$ & 12 & 7.609 & $a, b$ & 1996.11 & $10118.94-10133.90$ & 6 & 7.597 & $\mathrm{~b}$ \\
\hline 1989.47 & 7688.70-7707.70 & 13 & 7.602 & $a, b$ & 1996.18 & $10143.85-10155.84$ & 7 & 7.611 & $\mathrm{~b}$ \\
\hline 1990.01 & $7892.43-7903.03$ & 6 & 7.625 & $\mathrm{~b}, \mathrm{c}$ & 1996.22 & $10158.85-10166.90$ & 24 & 7.603 & $a, b$ \\
\hline 1990.08 & 7915.98-7926.95 & 7 & 7.625 & $\mathrm{~b}$ & 1996.25 & $10165.82-10181.87$ & 10 & 7.614 & $a, b$ \\
\hline 1990.17 & 7945.91-7958.87 & 6 & 7.625 & $\mathrm{~b}$ & 1996.32 & $10193.84-10208.84$ & 8 & 7.633 & $\mathrm{~b}$ \\
\hline 1990.29 & 7990.78-8007.81 & 14 & 7.603 & $\mathrm{~b}$ & 1996.37 & $10212.80-10226.72$ & 9 & 7.630 & $a, d$ \\
\hline 1990.36 & $8015.73-8029.78$ & 17 & 7.605 & $a, b$ & 1996.42 & $10233.74-10244.75$ & 19 & 7.632 & $\mathrm{~d}$ \\
\hline 1990.45 & $8045.73-8065.73$ & 14 & 7.601 & $\mathrm{a}, \mathrm{c}$ & 1996.47 & $10250.69-10261.70$ & 17 & 7.638 & $\mathrm{~d}$ \\
\hline 1991.06 & $8269.98-8288.34$ & 10 & 7.617 & $\mathrm{~b}, \mathrm{c}$ & 1996.98 & $10432.04-10451.01$ & 15 & 7.616 & $b, d$ \\
\hline 1991.20 & $8307.88-8348.77$ & 17 & 7.616 & $\mathrm{~b}, \mathrm{c}$ & 1997.07 & $10465.95-10482.00$ & 14 & 7.593 & $b, d$ \\
\hline 1991.27 & $8350.76-8361.73$ & 10 & 7.633 & $\mathrm{~b}$ & 1997.12 & $10485.89-10503.93$ & 28 & 7.606 & $b, d$ \\
\hline 1991.35 & $8370.73-8394.70$ & 25 & 7.613 & $a, b$ & 1997.17 & $10507.03-10514.90$ & 14 & 7.601 & $b, d$ \\
\hline 1991.40 & $8395.73-8405.72$ & 19 & 7.622 & $\mathrm{a}, \mathrm{b}, \mathrm{c}$ & 1997.22 & $10517.93-10537.87$ & 27 & 7.618 & $b, d$ \\
\hline 1991.42 & $8406.72-8415.71$ & 8 & 7.618 & $\mathrm{~b}$ & 1997.29 & $10546.72-10559.69$ & 21 & 7.639 & $\mathrm{~d}$ \\
\hline 1991.48 & $8422.76-8438.73$ & 10 & 7.600 & $\mathrm{a}, \mathrm{b}, \mathrm{c}$ & 1997.32 & $10562.69-10571.78$ & 13 & 7.643 & d \\
\hline 1992.20 & $8687.85-8705.82$ & 7 & 7.611 & $\mathrm{~b}$ & 1997.36 & $10573.71-10583.82$ & 7 & 7.655 & $\mathrm{~d}$ \\
\hline 1992.27 & $8718.82-8726.81$ & 5 & 7.610 & $\mathrm{~b}, \mathrm{c}$ & 1997.40 & 10590.69-10601.72 & 7 & 7.661 & d \\
\hline 1992.31 & $8731.85-8741.82$ & 11 & 7.606 & $a, b, c$ & 1997.45 & $10602.70-10620.69$ & 11 & 7.672 & d \\
\hline 1992.36 & 8749.84-8759.81 & 5 & 7.601 & $a, b$ & 1998.06 & $10823.00-10850.93$ & 15 & 7.649 & $\mathrm{~b}$ \\
\hline 1992.43 & $8774.46-8786.70$ & 8 & 7.601 & $a, b, c$ & 1998.24 & $10889.83-10914.75$ & 13 & 7.641 & $\mathrm{~b}$ \\
\hline 1992.48 & 8791.79-8805.70 & 8 & 7.599 & $\mathrm{a}, \mathrm{c}$ & 1998.34 & $10921.75-10949.65$ & 5 & 7.662 & $\mathrm{~b}$ \\
\hline 1993.09 & 9018.94-9029.97 & 4 & 7.584 & $a, b$ & 1998.42 & 10957.65-10977.66 & 6 & 7.642 & $\mathrm{~b}$ \\
\hline 1993.28 & 9077.79-9101.75 & 17 & 7.607 & $a, b$ & 1998.47 & 10979.67-10994.70 & 7 & 7.648 & $\mathrm{~b}$ \\
\hline 1993.33 & 9103.74-9114.72 & 14 & 7.614 & $a, b$ & 1999.04 & $11183.97-11201.91$ & 8 & 7.701 & $\mathrm{~b}$ \\
\hline 1993.37 & 9115.78-9132.66 & 25 & 7.620 & $a, b$ & 1999.11 & $11212.87-11229.91$ & 5 & 7.676 & $\mathrm{~b}$ \\
\hline 1993.42 & $9135.66-9144.73$ & 19 & 7.611 & $a, b$ & 1999.18 & $11232.86-11256.80$ & 8 & 7.674 & $\mathrm{~b}$ \\
\hline 1993.44 & $9145.67-9152.74$ & 19 & 7.615 & $a, b$ & 1999.23 & $11257.80-11266.77$ & 6 & 7.673 & $\mathrm{~b}$ \\
\hline 1993.47 & 9153.66-9166.66 & 11 & 7.612 & $a, b$ & 1999.30 & $11284.98-11294.70$ & 6 & 7.664 & $\mathrm{~b}$ \\
\hline 1994.09 & 9379.96-9393.92 & 11 & 7.603 & $\mathrm{~b}$ & 2000.02 & 11547.99-11560.96 & 9 & 7.679 & $\mathrm{~b}$ \\
\hline 1994.14 & 9399.90-9411.87 & 8 & 7.594 & $b, d$ & 2000.11 & $11578.91-11590.88$ & 5 & 7.675 & $\mathrm{~b}$ \\
\hline 1994.19 & $9416.85-9426.88$ & 23 & 7.599 & $b, d$ & 2000.24 & $11623.79-11640.75$ & 11 & 7.665 & $\mathrm{~b}$ \\
\hline 1994.26 & 9439.79-9456.74 & 19 & 7.610 & $b, d$ & 2000.30 & $11645.78-11659.70$ & 10 & 7.666 & $\mathrm{~b}$ \\
\hline 1994.31 & 9462.73-9471.79 & 19 & 7.594 & $\mathrm{~b}, \mathrm{~d}$ & 2000.34 & $11663.72-11675.79$ & 8 & 7.682 & $\mathrm{~b}$ \\
\hline
\end{tabular}

(a) Lockwood et al. (1997); (b) Villanova photometry; (c) Hipparcos; (d) Strassmeier et al. (1997, 1999).

\section{Discussion}

\subsection{Photospheric and chromospheric activity cycles}

The magnetic activity cycles and the longer-term trends that were found for our six targets are the first determined from photometric data. All the previous detections of variations in the long-term activity were based on the CaII H\&K fluxes, since our program stars are being observed at Mt. Wilson Observatory as part of the $H K$ project (Baliunas et al. 1995).

The results we obtained from photometric data partly differ from those based on the Ca II H\&K fluxes. In the following the results based on the two above mentioned different activity 


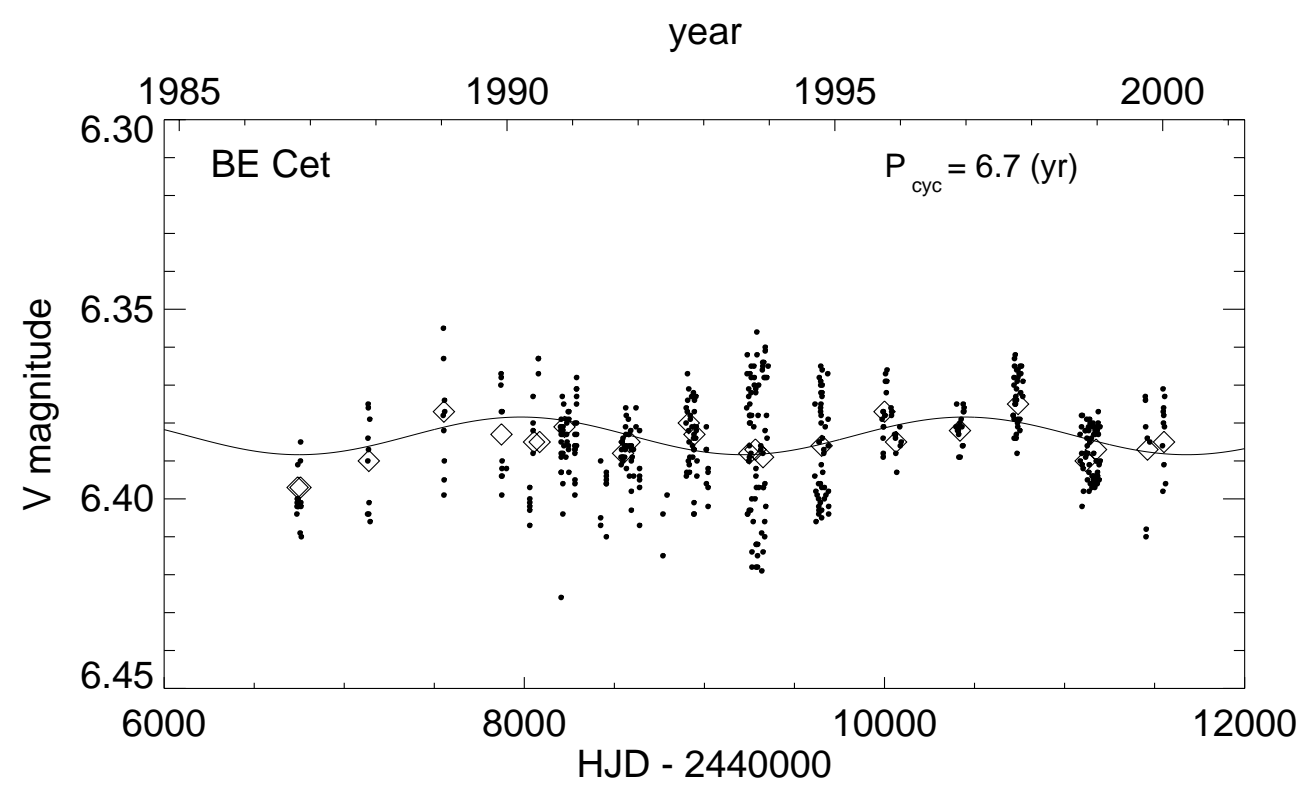

Fig. 4. BE Cet: time sequence of $V$-band magnitudes. Continuous line is a sinusoidal fit to the data with the period $P_{\text {cyc }}=6.7 \pm 0.7$ (yr). Open diamonds are the mean magnitudes computed from individual light curves $\left(V_{\max }+V_{\min }\right) / 2$ and used in the periodogram analysis.

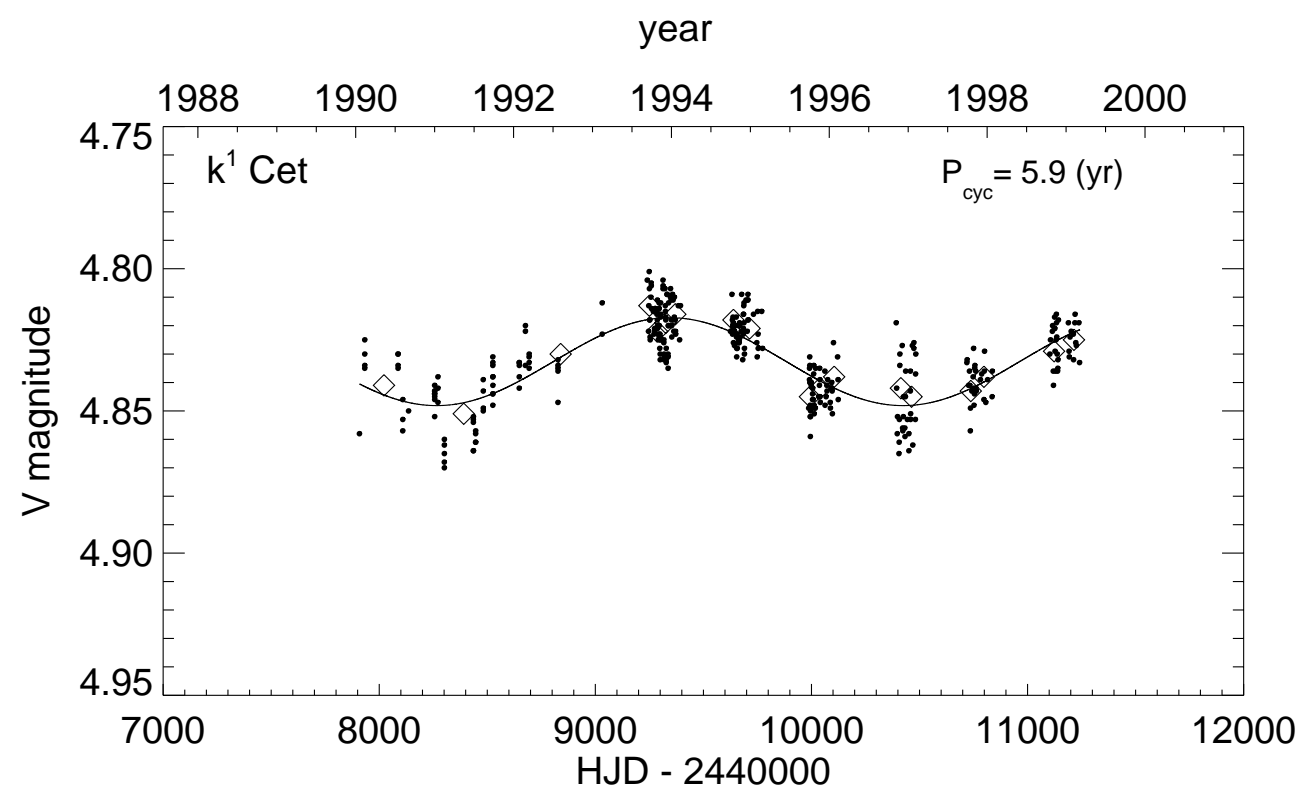

Fig. 5. $\mathrm{k}^{1}$ Cet: time sequence of $V$-band magnitudes. Continuous line is a sinusoidal fit to the data with a period $P_{\text {cyc }}=5.9 \pm 0.2$ (yr). Open diamonds have same the meaning as in Fig. 4.

diagnostics are compared and the differences discussed for each program star.

$\mathrm{BE}$ Cet has an average chromospheric cycle of $P_{\text {CaII }}=$ $9.1 \pm 0.3 \mathrm{yr}$ which is graded with a fair false alarm probability in the $10^{-5}-10^{-2}$ range. $\mathrm{BE}$ Cet also shows a longer-term trend. BE Cet has been also observed since 1984 within the photometric monitoring program carried out at the Lowell Observatory (Lockwood et al. 1997). Radick et al. (1998) found that the short- and long-term brightness variations of BE Cet are correlated to the CaII HK emission short- and long-term variations, respectively, with high $(p<0.05)$ significance level.

EK Dra is reported by Baliunas et al. (1995) with variable but not cyclic $\mathrm{Ca}$ II $\mathrm{H} \& \mathrm{~K}$ fluxes superimposed on a longer-term trend. It is also being observed at the Lowell Observatory and its short- and long-term photometric variations resulted to be highly correlated to the CaII HK emission variations.

Although to our knowledge the results of a period search in the photometric data collected at the Lowell Observatory has never been reported, however, the mentioned high degree correlations between photometric and $\mathrm{HK}$ emission variations would suggest that the same activity cycle found in the HK emission holds for the photometric data, that is, BE Cet is expected to show a starspot cycle of $P=9.1 \mathrm{yr}$ and EK Dra to show no starspot cycle but a longer-term trend.

As reported in Table 1 we found for BE Cet a cycle of $P_{\text {cyc }}=6.7 \mathrm{yr}$. It must be noted that the cycle period of BE Cet 


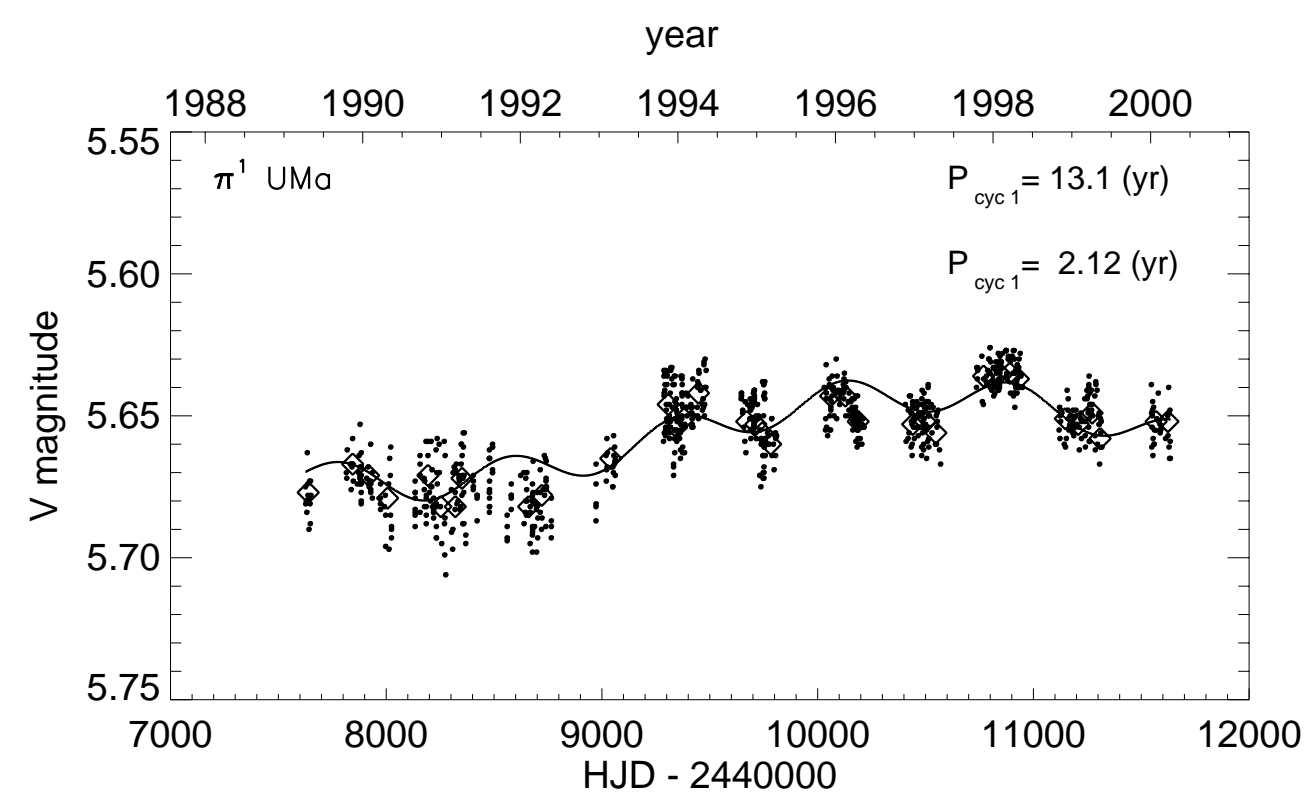

Fig. 6. $\pi^{1}$ UMa: time sequence of $V$-band magnitudes. Continuous line is a sinusoidal fit to the data with two periods: $P_{\text {cyc1 }}=13.1 \pm 0.9$ (yr) and $P_{\text {cyc1 }}=2.12 \pm 0.05$ (yr). Open diamonds have the same meaning as in Fig. 4 .

year

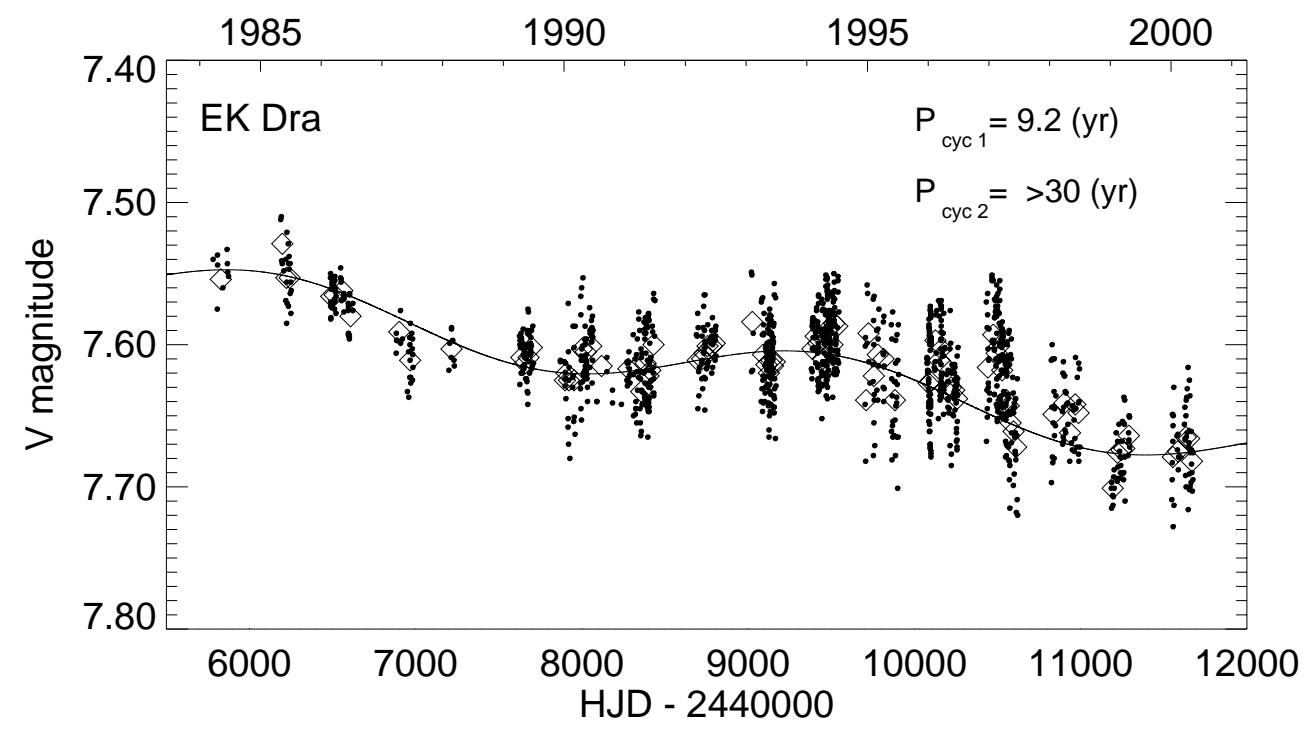

Fig. 7. EK Dra: time sequence of $V$-band magnitudes. Continuous line is a sinusoidal fit to the data with a period $P_{\text {cyc }}=9.2 \pm 0.4$ (yr) plus a linear long-term trend, which in the case of a cycle is expected to be longer than 30 years. Open diamonds have same the meaning as in Fig. 4.

was detected by our periodogram analysis with a FAP (0.41) significantly higher as compared to the other stars. Moreover, this cycle period is supported by both the rotation period seasonal variations and by the pooled variance analysis. In Paper II of this series dedicated to the SDR, we will show that the photometric rotation period of BE Cet undergoes year-to-year cyclic changes with a 6.7-yr period and also the pooled variance profile has a maximum at about the same time scale.

In the case of EK Dra we found a primary cycle of $P_{\text {cyc } 1}=$ $9.2 \mathrm{yr}$ plus a longer-term trend (which may be a segment of a cycle longer than $30 \mathrm{yr}$ if it is periodic).

The difference between the results we obtained and those which may be found from the analysis carried out by Radick et al. (1998) may arise mainly from the different extension and time location of the datasets. In fact, the periodogram results are sensitive to different time interval lengths and, moreover, the activity is not strictly periodic with possible small variations in amplitude and lengths from cycle to cycle. Finally, different observing approaches, more precisely, the constancy and frequency with which the targets have been monitored may contribute to get different results. The periodogram analyses presented in this paper are based on a larger number of observations and over a longer interval of time compared to the Lowell data set. We used 407 nights of observations over 13 years for BE Cet against 177 over 11 years at Lowell; we used 1262 nights of observations over 16 years for EK Dra against 244 over 11 years at Lowell. More importantly, the present analysis is based on a sequence of light curves which are well 


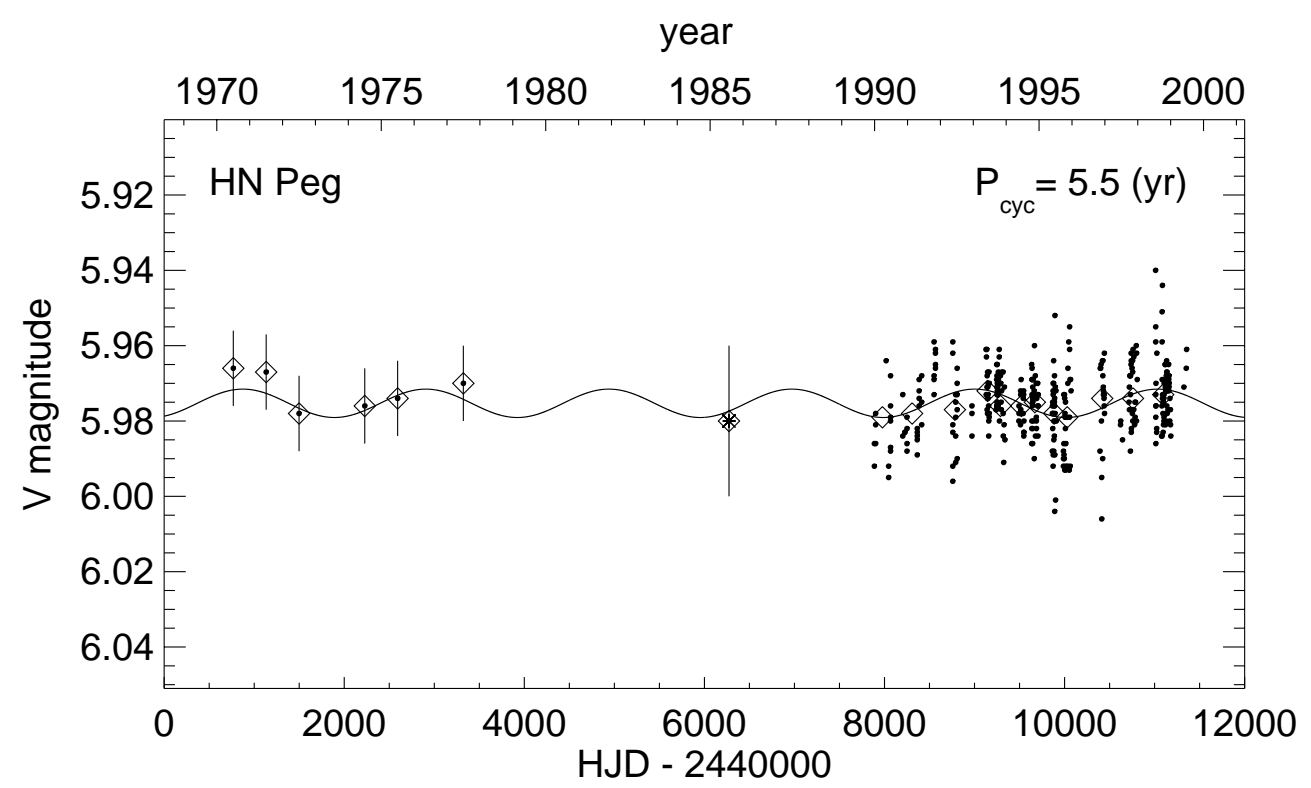

Fig. 8. HN Peg: time sequence of $V$-band magnitudes. Continuous line is a sinusoidal fit to the data with a period $P_{\text {cyc }}=5.5 \pm 0.3$ (yr). Open diamonds have the same meaning as in Fig. 4. The vertical bars indicate the peak-to-peak light curves amplitudes taken from Blanco et al. (1972, 1979) and Reglero (1986).

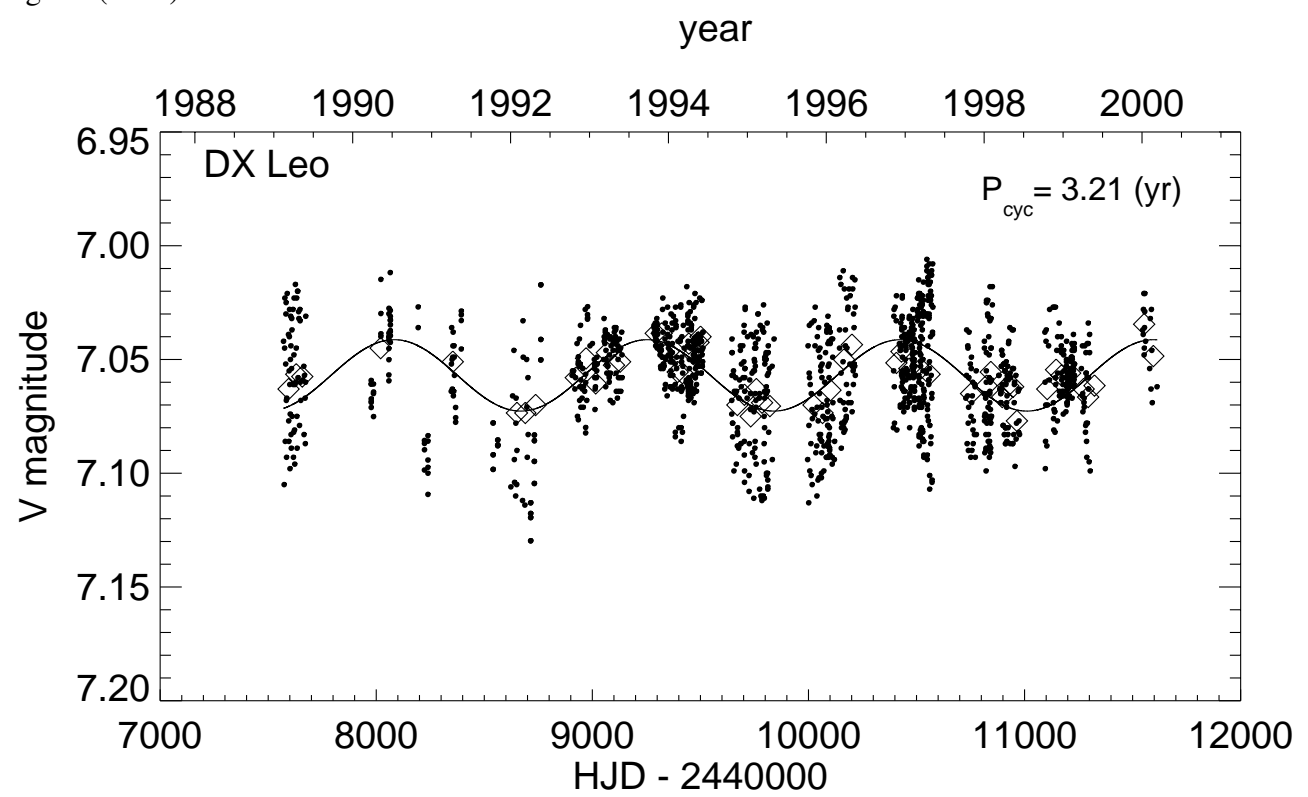

Fig. 9. DX Leo: time sequence of $V$-band magnitudes. Continuous line is a sinusoidal fit to the data with a period $P_{\text {cyc }}=3.21 \pm 0.05$ (yr). Open diamonds have the same meaning as in Fig. 4.

covered in phase. This guarantees the determination of reliable seasonal mean magnitudes and, consequently, more accurate detection and estimates of possible cycles. Unfortunately, 8-10 observations per season, or even less in seasons with poor weather, were collected at the Lowell Observatory (see Lockwood et al. 1997). The mean magnitudes computed from those data may differ from the star's actual mean brightness. In spite of the different results we found, we may expect that the correlation with the HK emission for BE Cet and EK Dra still exists but with a lower level of significance.

It must be noted that Dorren \& Guinan (1994) first suggested the existence of a possible activity cycle of EK Dra of about 12 years from the 1982-1994 photometric dataset. The existence of such solar-like cycle, which was questioned by
Saar \& Brandenburg (1999) on the basis of the Radick et al.'s analisys (1998), is now re-confirmed, even if this time it is estimated with a much more increased accuracy.

$\mathrm{k}^{1}$ Cet has an average chromospheric cycle length of $P_{\text {CaII }}=5.6 \pm 0.1 \mathrm{yr}$ with a false alarm probability graded "fair" in the $10^{-5}-10^{-2}$ range and with a longer-term trend (Baliunas et al. 1995). The photometric cycle we found is similar in length $\left(P_{\text {cyc }}=5.9 \mathrm{yr}\right)$ and $F A P$, but no evidence of longer-term trend was found.

$\pi^{1}$ UMa is reported by Baliunas et al. (1995) to have variable CaII $\mathrm{HK}$ emission but with no definite periodicity. However, it must be noted that their periodogram analysis was based on a 7-yr time interval. On the contrary, on a longer 
time basis of 11 years we found two cycles $\left(P_{\mathrm{cyc} 1}=13.1 \mathrm{yr}\right.$, $P_{\text {cyc } 2}=2.12$ yr) both with a fair confidence level.

HN Peg has a chromospheric cycle of $P_{\text {CaII }}=6.2 \pm$ $0.2 \mathrm{yr}$ with a false alarm probability graded "poor" in the $10^{-2}-10^{-1}$ range and with a possible longer-term trend (Baliunas et al. 1995). We found a cycle of $P_{\text {cyc }}=5.48 \mathrm{yr}$ with a good confidence level.

DX Leo has a chromospheric cycle of $P_{\text {CaII }}=2.8 \pm$ $0.1 \mathrm{yr}$ with a false alarm probability graded "poor" in the $10^{-2}-10^{-1}$ range. However, even in this case the periodogram analysis was based on a relatively short time interval of 5 years. On the contrary, from a longer time base of 12 years we found a cycle of $P_{\text {cyc }}=3.21 \mathrm{yr}$ with a good confidence level. This cycle length updates the value reported in Messina et al. (1999), whose analysis was carried out on a smaller database of 10 years and taking into consideration only the Villanova photometry.

The differences between the long-term behaviour (cyclic and/or long-term trend) as inferred from the photometric data or from the CaII HK emission fluxes depend, as mentioned, mainly on the different time extension and location of the dataset taken into consideration. However, intrinsic differences i.e. differences due to the different nature of the activity indicators: photospheric dark spots in the present analysis and chromospheric bright plages in the Baliunas et al. (1995) analysis can not be excluded.

If we consider for example the solar case, during Cycles 20-22 the Sun had a chromospheric cycle of $P_{\text {CaII }}=$ $10.0 \pm 0.1 \mathrm{yr}$ (Baliunas et al. 1995), which slightly differed from its starspots cycle of $P_{\text {cyc }}=10.5 \pm 0.1 \mathrm{yr}$. The sunspot cycle was determined by us using the Royal Greenwich Observatory data archive.

Moreover, the existence of a range in the correlation degree between the photometric brightness and HK emission variations found by Radick et al. (1998) goes into the same direction, that is of a not strict time correspondence of the activity levels at different atmospheric layers.

\subsection{Correlations with stellar parameters}

The relationships between the frequency $\omega_{\text {cyc }}$ of magnetic activity cycle, the rotational frequency $\Omega$ and other global stellar properties have been explored for large samples of stars including dwarfs, evolved stars, active binaries and the results have been presented in several papers (e.g. see Ossendrijver 1997; Brandenburg et al. 1998; Saar \& Brandenburg 1999; Lanza \& Rodonò 1999; Oláh et al. 2000). In the following we refer to the empirical relations found by Saar \& Brandenburg (1999) which are derived from the most complete star sample to date. The cycles on which these studies are based are derived from CaII HK fluxes, photometry, and orbital period changes. In a $\omega_{\text {cyc }} / \Omega-R_{0}^{-1}$ diagram (where $R_{0}$ is the Rossby number) stars tend to concentrate along three branches with different slopes. In Fig. 1 we plot the three branches as computed by Saar \& Brandenburg (1999) (cf. their Fig. 5). More active stars tend to lie on the lower branch ("active"), while less active stars in the upper ("inactive"). A few stars have double cycles falling on both branches. The most active stars populate a third branch with opposite slope ("super-active"). Moreover, a possible transition zone between the super-active and active branches may exist.

In Fig. 1 we plot our new estimates of cycle lengths from the photometry. To compute the inverse Rossby number $\left(R_{0}^{-1}\right)$ we used the revised convective turnover time $\left(\tau_{\mathrm{c}}\right)$ derived by Saar (1998). Beside the Sun, two other single main-sequence stars exist to our knowledge with magnetic activity cycles estimated exclusively from photometric data which are not included in The Sun in time project: LQ Hya, a single K2V star with a rotation period of $P_{\text {rot }}=1.61^{\mathrm{d}}$ and multiple starspot cycles $P_{\mathrm{cyc} 1}=6.75 \mathrm{yr}, P_{\mathrm{cyc} 2}=11.40 \mathrm{yr}$ and $P_{\mathrm{cyc} 3}=2.8 \mathrm{yr}$ (Oláh et al. 2000) and AB Dor, a single K0V star with a rotation period of $P_{\text {rot }}=0.541^{\mathrm{d}}$ and a primary starspots cycle $P_{\text {cyc1 }}=18.30 \mathrm{yr}$ and a secondary cycle $P_{\text {cyc } 2}=5.9 \mathrm{yr}$ (Messina et al. in progress). These stars have been added to our sample for the following analysis. It must be pointed out that our final sample contains only single main-sequence stars, while the branches are computed from an expanded sample containing also binaries and out of main-sequence stars.

All the cycle lengths, except for EK Dra, fit well the empirical relations with global stellar parameters derived from larger stellar samples. While the fastest rotating stars (AB Dor and LQ Hya) fall in the super-active branch and in the transition zone, respectively, the slower rotating stars are all on the active branch. The only exception is represented by EK Dra. Its primary cycle of $P=9.2 \mathrm{yr}$ appears where apparently no cycle is expected from the empirical relation, while its longer-term trend, if segment of a cycle longer than 30 years, may fall in the extension of the super-active branch. Also the secondary period of AB Dor apparently lies above the "super-active" branch.

In Fig. 2 we investigate on the dependence of the activity cycle over the rotational frequency vs. age. Although only nine stars are included in our sample, it appears that the range in the primary cycle length converges in time from a maximum dispersion at the Pleiades age $(0.13 \mathrm{Gyr})$ towards the 11 -yr solar cycle at an age of $\simeq 4.6 \mathrm{Gyr}$.

\subsection{The cycle amplitude}

The correlations between the amplitude of the overall $V$-band variability and the global stellar parameters have been also investigated. The highest degree of correlation is found with the inverse Rossby number as shown in Fig. 3. The overall $V$-band variability amplitude at any given epoch arises primarily from the contribution from spotted regions evenly and unevenly distributed in longitude (the last being responsible for the light curve amplitude). In Fig. 3 we considered the difference $(\Delta V)$ between the observed brightest and faintest magnitudes as measure of the capability of the stars to produce the largest spotted magnetic regions (the maximum total spotted area).

The maximum light amplitude of such long-term overall variability should be considered, at any rate, as a lower limit since the observed brightest magnitude may not correspond to the actual unspotted magnitude (Neff et al. 1995). For example, the presence of uniformly distributed spotted regions at 
Table 8. DX Leo.

\begin{tabular}{|c|c|c|c|c|c|c|c|c|c|}
\hline Mid epoch & HJD range & $N_{\mathrm{m}}$ & $V_{\text {mean }}$ & Source & Mid epoch & HJD range & $N_{\mathrm{m}}$ & $V_{\text {mean }}$ & Source \\
\hline 1989.19 & $7570.82-7617.69$ & 33 & 7.063 & $\mathrm{a}$ & 1995.83 & $9996.02-10050.88$ & 31 & 7.070 & $\mathrm{~b}$ \\
\hline 1989.28 & $7618.80-7640.70$ & 14 & 7.056 & a & 1995.95 & $10051.90-10078.84$ & 18 & 7.073 & $\mathrm{~b}$ \\
\hline 1989.36 & $7641.65-7673.66$ & 13 & 7.057 & a & 1996.05 & 10082.79-10120.84 & 44 & 7.063 & $a, b$ \\
\hline 1991.27 & $8346.74-8361.70$ & 12 & 7.051 & a & 1996.22 & $10143.73-10184.73$ & 29 & 7.050 & $\mathrm{~b}, \mathrm{c}$ \\
\hline 1992.08 & $8540.72-8761.57$ & 26 & 7.073 & $\mathrm{a}$ & 1996.32 & $10185.72-10216.71$ & 18 & 7.043 & $\mathrm{~b}$ \\
\hline 1992.19 & $8676.75-8702.71$ & 8 & 7.073 & $\mathrm{~b}$ & 1996.88 & 10392.92-10420.87 & 10 & 7.051 & $\mathrm{~b}$ \\
\hline 1992.82 & $8906.01-8940.95$ & 13 & 7.058 & $\mathrm{~b}$ & 1996.95 & $10423.92-10436.81$ & 24 & 7.046 & $\mathrm{~b}, \mathrm{c}$ \\
\hline 1992.95 & 8941.95-8999.94 & 15 & 7.049 & $\mathrm{~b}$ & 1997.05 & $10440.80-10492.84$ & 81 & 7.054 & $a, b, d$ \\
\hline 1993.07 & $9009.02-9021.88$ & 13 & 7.060 & $\mathrm{~b}$ & 1997.19 & $10503.92-10534.85$ & 67 & 7.054 & $\mathrm{a}, \mathrm{b}, \mathrm{d}$ \\
\hline 1993.22 & $9051.82-9082.73$ & 22 & 7.047 & $a, b$ & 1997.31 & $10545.71-10575.69$ & 53 & 7.056 & $b, d$ \\
\hline 1993.31 & 9082.73-9117.68 & 21 & 7.052 & $a, b$ & 1997.83 & $10728.01-10776.90$ & 29 & 7.065 & $\mathrm{~b}$ \\
\hline 1993.39 & 9118.68-9137.65 & 10 & 7.051 & $\mathrm{~b}$ & 1998.02 & $10811.87-10836.87$ & 32 & 7.061 & $a, b$ \\
\hline 1993.83 & 9284.96-9301.95 & 16 & 7.038 & $\mathrm{a}, \mathrm{b}$ & 1998.08 & 10837.02-10850.99 & 23 & 7.055 & $\mathrm{~b}, \mathrm{e}$ \\
\hline 1993.91 & 9311.93-9329.87 & 16 & 7.038 & $\mathrm{a}, \mathrm{b}$ & 1998.23 & $10880.88-10911.82$ & 16 & 7.066 & $\mathrm{~b}$ \\
\hline 1994.00 & 9331.91-9374.81 & 32 & 7.046 & $\mathrm{~b}$ & 1998.30 & 10912.72-10935.66 & 18 & 7.061 & $\mathrm{~b}$ \\
\hline 1994.11 & 9382.83-9406.87 & 14 & 7.055 & $\mathrm{~b}$ & 1998.37 & 10942.71-10951.68 & 8 & 7.062 & $\mathrm{~b}$ \\
\hline 1994.18 & 9408.75-9424.76 & 15 & 7.056 & $\mathrm{~b}$ & 1998.42 & $10952.69-10976.65$ & 10 & 7.077 & $\mathrm{~b}$ \\
\hline 1994.26 & $9433.71-9458.67$ & 38 & 7.043 & $\mathrm{a}, \mathrm{b}, \mathrm{c}$ & 1998.80 & 11094.00-11115.95 & 11 & 7.063 & $\mathrm{~b}$ \\
\hline 1994.31 & $9462.72-9470.70$ & 10 & 7.047 & $\mathrm{a}, \mathrm{b}$ & 1998.91 & $11131.92-11159.85$ & 16 & 7.054 & $\mathrm{~b}$ \\
\hline 1994.38 & $9471.71-9511.65$ & 43 & 7.042 & $\mathrm{a}, \mathrm{b}, \mathrm{c}$ & 1998.98 & $11161.05-11181.85$ & 17 & 7.057 & $\mathrm{~b}$ \\
\hline 1994.41 & $9489.67-9511.65$ & 15 & 7.040 & $\mathrm{~b}$ & 1999.08 & $11191.03-11230.95$ & 66 & 7.056 & $\mathrm{~b}, \mathrm{e}$ \\
\hline 1994.87 & 9644.97-9696.97 & 32 & 7.070 & $a, b$ & 1999.26 & $11262.73-11284.72$ & 12 & 7.063 & $\mathrm{~b}$ \\
\hline 1994.96 & 9700.83-9707.00 & 12 & 7.065 & $\mathrm{~b}$ & 1999.32 & $11284.72-11303.70$ & 14 & 7.066 & $\mathrm{~b}$ \\
\hline 1995.04 & 9715.89-9749.94 & 16 & 7.075 & $a, b$ & 1999.40 & $11304.69-11342.65$ & 26 & 7.061 & $\mathrm{~b}$ \\
\hline 1995.11 & 9750.77-9766.78 & 16 & 7.063 & $a, b$ & 2000.02 & $11546.91-11559.88$ & 9 & 7.034 & $\mathrm{e}$ \\
\hline 1995.19 & 9772.79-9797.87 & 21 & 7.069 & $a, b$ & 2000.14 & $11578.88-11612.79$ & 6 & 7.048 & $\mathrm{e}$ \\
\hline 1995.29 & 9802.74-9840.69 & 23 & 7.070 & $a, b$ & & & & & \\
\hline
\end{tabular}

(a) Messina et al. (1999); (b) Gaidos et al. (2000); (c) Strassmeier et al. (1997); (d) Strassmeier et al. (1999);

(e) Villanova photometry.

the epoch of the observed brightest magnitude would not be revealed by the $V$-band photometry. However, because of the large number of available light curves and of the extended time sequence for each program star we are confident that the observed amplitude can reliably approach the actual value (see e.g. Messina et al. 2001). The existence of such high degree correlation between the maximum amplitude of the long-term variability and the inverse Rossby number indicates that the stellar rotation controls both the short-term (as already known, see e.g. Messina et al. (2001) and long-term areal coverage of starspots. In Messina et al. (2001) it is shown that the increase in the light curve peak-to-peak amplitude with rotation rate levels off at the fastest rotation rates, which may be interpreted as the evidence for saturation in the starspot coverage. It is interesting to note from the cubic fit to the data shown in Fig. 3 that also the overall variability (uniformly + unevenly distributed spotted areas) levels off at high rotation rates, which are represented in the present analysis by LQ Hya and AB Dor. Such behaviour may suggest a nearly total filling of the photosphere by spotted regions during the cycle activity maximum in the fastest rotators. If this is the case, the resulting scenario is certainly different from the dynamo wave which migrates in latitude over the activity cycle but produces spots that cover only a small fraction of the photosphere even at the activity maximum as observed in the Sun.

Another relevant result, which will be better addressed in a later paper, is that due to the almost completely (and therefore uniform) filling of the photosphere by spotted regions, low amplitude light curves are expected to be observed around the activity maximum, which is clearly not the case at least from this study (see Fig. 4 to Fig. 9.)

In the cited relations EK Dra plays again a peculiar role. If we consider the amplitude of the 9.2-yr cycle (filled circle in Fig. 3), it fits quite well the $\Delta V-R_{0}^{-1}$ relation. However, when the amplitude of the longer-term trend is also included (asterisk in Fig. 3) it lies significantly above the curve. If the long-term trend is not a segment of an unresolved longer cycle, we may think that stellar rotation controls only the amplitude of the cycles and it is not apparently related to the trends. If EK Dra turns out to have multiple starspot cycles, its behaviour is clearly unusual. 


\section{Conclusions}

The ground-based observations carried out as part of The Sun in Time project have now become sufficiently extended in time to enable the study of the photospheric magnetic activity on time scales comparable to the 11-yr solar cycle. From the analysis discussed here some remarkable results can be inferred:

- The existence of solar-like starspot cycles has been firmly established for six young solar analogues. The presence of multiperiodic cycles as well as of long-term trends have been also detected.

- Some differences are found with respect to the results based on long-term CaII H\&K emission fluxes measurements, which can be likely attributed to both differences in time location and length of the datasets, but also from the intrinsic properties of the activity tracers used, that is dark photospheric spots and bright chromospheric plages.

- The dependence of the activity cycle length over rotation frequency on the inverse Rossby number agrees with those found for larger samples containing also binary and postmain-sequence stars. Only EK Dra seems to be unusual lying its cycle length above the cited relations;

- The range in the cycle period observed among the Pleiadesage stars of the sample seems to converge in time similar in length to the 11-yr solar cycle.

- The overall photometric variability appears highly correlated with the inverse Rossby number, suggesting that the stellar rotation controls the level of magnetic activity in photosphere even over the longest time scales. The levelling-off (see Fig. 3) observed at the highest rotation rates may be interpreted as arising from a stellar photosphere nearly covered by spots during the maximum of activity. This is a different scenario from the migrating wave observed in the solar case. However, such an interpretation may be in contradiction with the level of asymmetry of the spotted regions as inferred from the large amplitude of the light curves around the maximum of activity.

Acknowledgements. The authors are very grateful to the Dr. G.W. Lockwood for providing us with the Lowell photometric data of EK Dra. The extensive use of the SIMBAD and ADS databases operated by the CDS center, Strasbourg, France, is also gratefully acknowledged.

Active star research at the Catania Astrophysical Observatory is funded by MURST (Ministero dell'Università e della Ricerca Scientifica e Tecnologica) and the Regione Siciliana, whose financial support is gratefully acknowledged. This research is also supported by the U.S. National Science Foundation for Research whose financial support is gratefully acknowledged.

\section{References}

Baliunas, S.L., Donahue, R. A., Soon, W.H., et al. 1995, ApJ, 438, 269

Bessel, M. S. 1990, PASP, 102, 1181

Blanco, C., Catalano, S., \& Godoli, G. 1972, Mem. SAIt, 43, 663

Blanco, C., Catalano, S., \& Marilli, E. 1979, A\&AS, 36, 297

Bochanski, J. J., Guinan, E. F., DePasquale, J. M., \& McCook, G.P. 2001, IBVS, 5043

Boyd, L. J., Genet, R. M., \& Hall, D. S. 1984, IAPPP Comm., 15, 20

Brandenburg, A., Saar, S. H., \& Turpin, C. J. 1998, ApJ, 498, L51

Chugainov, P. F. 1980, Iz. Kry., 61, 127

Cutispoto, G. 1991, A\&AS, 89, 435

Cutispoto, G. 1992, A\&AS, 95, 397

Cutispoto, G. 1995, A\&AS, 111, 507

Dobson, A. K., Donahue, R. A., Radick, R. R., \& Kadlec, K. L. 1990, in The Sixth Cambridge Symposium on Cool Stars, Stellar Systems and the Sun, ed. G. Wallerstein, ASP Conf. Ser., 9, 132

Donahue, R. A., Saar, S. H., \& Baliunas, S. L. 1996, ApJ, 466, 384

Dorren, J. D., \& Guinan, E. F. 1994, ApJ, 428, 805

Duquennoy, A., \& Mayor, M. 1991, A\&A, 248, 485

Frasca, A., Freire Ferrero, R., Marilli, E., \& Catalano, S. 2000, A\&A, 364,179

Gaidos, E. J., Henry, G. W., \& Henry, S. M. 2000, AJ, 120, 1006

Guedel, M., Guinan, E. F., \& Skinner, S. L. 1997, ApJ, 483, 947

Guinan, E. F., \& McCook, G. P. 1991, BAAS, 23, 874

Guinan, E. F., Ribas, I., \& Harper, G. M. 2001, AAS, 199, 8805

Henry, G. W., Fekel, F. C., \& Hall, D. S. 1995, AJ, 110, 2926

Horne, J. H., \& Baliunas, S. L. 1986, ApJ, 302, 757

Lanza, A. F., \& Rodonò, M. 1999, A\&A, 349, 887

Lockwood, G. W., Skiff, B. A., \& Radick, R. R. 1997, ApJ, 485, 789

Maulik, D., Donahue, R. A., \& Baliunas, S. L. 1997, SAO Tech. Rep

Messina, S., Guinan, E. F., Lanza, A. F., \& Ambruster, C. 1999, A\&A, 347, 249

Messina, S., Rodonò, M., \& Guinan, E. F. 2001, A\&A, 366, 215

Neff, J. E., O’Neal, D., \& Saar, S. H. 1995, ApJ, 452, 879

Oláh, K., Kolláth, Z., \& Strassmeier, K. G. 2000, A\&A, 356, 643

Olsen, E. H. 1983, A\&AS, 54, 55

Ossendrijver, A. J. H. 1997, A\&A, 323, 151

Perryman, M. A. C. and The Hipparcos Science Team 1997, ESA SP-1200, vol. 1-12 (ESA Publ. Division, c/o ESTEC, Noordwijk, The Netherlands)

Radick, R. R., Lockwood, G. W., Skiff, B. A., \& Baliunas, S. L. 1998, ApJS, 118, 239

Reglero, V., Fabregat, J., \& Gomez de Castro, A. 1986, IBVS, 2904

Saar, S. H. 1998, in Coll Stars, Stellar Systems and the Sun, ed. R. A. Donahue, \& J. A. Bookbinder (Sam Francisco), ASP Conf. Ser., 154,211

Saar, S. H., \& Barndenburg, A. 1999, ApJ, 524, 295

Scargle, J. D. 1982, ApJ, 263, 835

Seeds, M. A. 1995, in Robotic Telescopes: current capabilities, present developements and future prospects for automated astronomy, ed. G. W. Henry, \& J. A. Eaton, PASPC, 79, 11

Stepień, K., \& Geyer, E. 1996, A\&AS, 117, 83

Strassmeier, K. G., Bartus, J., Cutispoto, G., \& Rodonò, M. 1997, A\&AS, 125, 11

Strassmeier, K. G., Serkowitsch, E., \& Granzer, T. 1999, A\&AS, 140, 29

Strömgren, B. 1966, ARAA, 4, 433

Wilson, O. C. 1953, General catalogue of Stellar Radial Velocities (Carnegie Inst. Washington Publ. 601) 